\title{
A new leader-based multicast scheme with a Raptor code in IEEE 802.11 multi-rate WLANs
}

\author{
Dongwan Kim, Noureddine Boudriga ${ }^{2}$ and Sunshin $\mathrm{An}^{1^{*}}$
}

\begin{abstract}
Multimedia streaming using a multicast scheme over IEEE 802.11 multi-rate wireless local area networks (WLANs) faces many challenges owing to the heterogeneous channel conditions of receivers. Although many multicast schemes have been proposed to support multimedia streaming over multi-rate WLANs, those schemes do not deal with multicast transmission efficiently. In this paper, a new leader-based multicast scheme with a Raptor code is proposed. The proposed scheme consists of a leader selection block, a scalable video coding (SVC) block with unequal error protection (UEP), and a link adaptation block with a Raptor code. In the proposed scheme, one of the multicast group members is selected as a multicast group leader according to a leader cost, and then link adaptation operates between the multicast transmitter and the multicast group leader. In addition, SVC, UEP, and Raptor code mechanisms are applied to guarantee the minimum required performance of multicast group members. The proposed scheme aims to enhance the link utilization and guarantees the minimum quality-of-service requirement of multicast group members. Simulation results show that the proposed scheme performs better than previous multicast schemes.
\end{abstract}

Keywords: Leader-based multicast; Raptor code; Link adaptation; Unequal error protection; IEEE 802.11 multi-rate WLANs

\section{Introduction}

IEEE 802.11 wireless local area networks (WLANs) have been successfully deployed in many indoor and outdoor environments. Although many wireless access network technologies, such as wideband code division multiple access (WCDMA) and long term evolution (LTE), have been standardized for wireless communication, IEEE 802.11 WLANs have still been the dominant wireless access technology owing to their low cost and easy deployment. The IEEE 802.11 working group specified several IEEE 802.11 WLAN standards, such as IEEE $802.11 \mathrm{a} / \mathrm{b} / \mathrm{g} / \mathrm{h} / \mathrm{aa} / \mathrm{ac}$, as their maximum data rate and target purpose. For throughput enhancement, IEEE $802.11 \mathrm{a} / \mathrm{b} / \mathrm{g} / \mathrm{h} / \mathrm{ac}$, which support multi-rate transmission through adaptive modulation and coding (AMC), were suggested [1]. Unlike other standards, IEEE 802.11aa suggests for the efficient transmission of unicast/multicast multimedia data [2].

\footnotetext{
* Correspondence: sunshin@dsys.korea.ac.kr

${ }^{1}$ The School of Electrical Engineering, Korea University, Anam-dong, Seongbuk-Gu, Seoul 136-701, South Korea

Full list of author information is available at the end of the article
}

In addition, as the demand for multimedia transmission increases, IEEE 802.11 WLANs are becoming to be regarded as an attractive and cost-effective method to deliver multimedia data to multiple nodes [3]. This can be achieved in three ways, unicast, multicast, and broadcast transmission. In the unicast case, multimedia data delivery to multiple nodes requires a significant amount of bandwidth when the number of nodes is high since the transmitter has to send a data packet to each and every node. In contrast, multicast and broadcast transmission use fixed channel bandwidths regardless of the number of nodes, thereby improving network utilization.

However, multicast transmission is less efficient in multi-rate WLANs since link adaption cannot be applied. This is because the IEEE 802.11 standards do not support feedback mechanisms such as acknowledgement (ACK) frame which is essential for link adaptation [4]. Consequently, according to IEEE 802.11 multi-rate WLAN standards such as IEEE $802.11 \mathrm{a} / \mathrm{b} / \mathrm{g} / \mathrm{n} / \mathrm{ac}$, all frames of multicast transmissions must be transmitted at one of the rates included in the basic transmission rate set - (i.e., lower transmission rates) - due to the absence

\section{实}

(C) 2014 Kim et al.; licensee Springer. This is an Open Access article distributed under the terms of the Creative Commons Attribution License (http://creativecommons.org/licenses/by/2.0), which permits unrestricted use, distribution, and reproduction in any medium, provided the original work is properly credited. 
of a feedback mechanism for estimating the channel state of multiple receivers. Moreover, the multicast in WLANs cannot guarantee reliability and quality of service $(\mathrm{Q} o \mathrm{~S})$ of a multimedia transmission due to absence of ACK frame.

Several solutions have been proposed to provide multimedia transmission over IEEE 802.11 WLANs efficiently by using a multicast scheme. In [5], an access point (AP) sets a leader in the group of multicast receivers, and an automatic retransmission request (ARQ) protocol is applied between the leader and the AP. A particular level of reliability is likely to be preserved for all multicast receivers in the group, if the node that has the lowest error probability is selected as the leader. In addition, a rate adaptation scheme with multicast in WLAN has been proposed in [6,7]. In [6], the transmission data rate is adapted to the user with the lowest signal-to-noise ratio (SNR) and, in [7], the channel conditions of all the users in the multicast group are reported for rate adaptation. In both schemes, additional signaling is required to obtain the SNR of all the receivers. Also, Villalon et al. [8] proposed a cross-layer approach for adaptive video multicast that considers the multi-rate capabilities of wireless networks.

Other solutions for multicast in IEEE 802.11 WLANs are based on a fountain code and scalable video coding (SVC). The fountain code and SVC are used for guaranteeing scalability and a minimum required QoS for different users. In [9], a joint SVC block with an unequal error protection (UEP) scheme was proposed to deal with heterogeneous wireless receivers. In addition, Choi et al. proposed a Raptor code-based video multicast transmission scheme that is a joint adaptation of the Raptor code rate and the physical (PHY) rate [10]. However, these schemes [6-10] do not optimize channel usage when transmitting multicast traffic over multi-rate WLANs, because they focus on the multicast receiver that has the worst channel condition.

In this paper, we propose a new leader-based multicast scheme with a Raptor code in IEEE 802.11 multi-rate WLANs. By applying the proposed scheme, mobile nodes can utilize wireless channel capacity efficiently, with a minimum required QoS in a noisy wireless channel environment guaranteed. Unlike existing schemes, the proposed scheme selects a leader node based on leader cost, rather than the worst channel condition node. Thus the proposed scheme performs link adaptation in a better channel condition, resulting in increased total system throughput. Also, the minimum required QoS is guaranteed by compensation methods such as SVC, UEP, and Raptor code schemes.

The rest of this paper is organized as follows. Related work is introduced in Section 2, the details of the proposed scheme are specified in Section 3, performance evaluation is described in Section 4, and conclusions are drawn in Section 5.

\section{Related works}

\subsection{Multi-rate wireless LAN}

The IEEE $802.11 \mathrm{a} / \mathrm{b} / \mathrm{g} / \mathrm{n} / \mathrm{ac}$ standards support multi-rate transmission through AMC. For example, the IEEE 802.11a PHY provides eight PHY modes, listed in Table 1, and the transmission rate can be changed from 6 to $54 \mathrm{Mbps}$, according to the channel state. The criterion for selecting transmission rate is receiver channel state, which is measured using a feedback mechanism. Measurement of receiver channel states is an essential procedure for proper utilization of wireless channel capacity in multirate transmission.

In general, rate adaptation mechanisms have been widely investigated and deployed for unicast transmission. In auto rate fallback (ARF) [11], a transmitter determines a proper transmission rate and then selects the next transmission rate based on the previous packet success transmission history on the transmitter side. The transmitter selects the next higher transmission rate, when it continuously receives an ACK frame from the receiver, and then selects a lower transmission rate, when continuous transmission errors are detected. While ARF estimates channel state information on the transmitter side, the other algorithm estimates the channel state on the receiver side to measure the channel states more precisely. In receiver-based auto rate (RBAR) [12], the transmitter and the receiver exchange request-to-send (RTS) and clear-to-send (CTS) frames prior to data transmission. The receiver estimates the channel conditions using an RTS, selects the best transmission rate, and notifies the transmitter using a CTS. However, the existing rate adaptation mechanisms $[11,12]$ for unicast transmission cannot be applied directly to multicast transmission owing to absence of a feedback mechanism in a multicast scheme. In a multicast scheme, the sender does not receive any channel information from receivers. Therefore, the transmitter cannot know the channel state of every receiver.

\begin{tabular}{lllll}
\multicolumn{5}{c}{ Table $\mathbf{1}$ Eight PHY modes of the IEEE 802.11a PHY } \\
\hline Mode & Data rate & Modulation & Code rate & Receiver sensitivity \\
\hline 1 & $6 \mathrm{Mbps}$ & BPSK & $1 / 2$ & $-90 \mathrm{dBm}$ \\
2 & $9 \mathrm{Mbps}$ & BPSK & $3 / 4$ & $-89 \mathrm{dBm}$ \\
3 & $12 \mathrm{Mbps}$ & QPSK & $1 / 2$ & $-87 \mathrm{dBm}$ \\
4 & $18 \mathrm{Mbps}$ & QPSK & $3 / 4$ & $-85 \mathrm{dBm}$ \\
5 & $24 \mathrm{Mbps}$ & 16-QAM & $1 / 2$ & $-82 \mathrm{dBm}$ \\
6 & $36 \mathrm{Mbps}$ & 16-QAM & $3 / 4$ & $-78 \mathrm{dBm}$ \\
7 & $48 \mathrm{Mbps}$ & 64-QAM & $2 / 3$ & $-72 \mathrm{dBm}$ \\
8 & $54 \mathrm{Mbps}$ & 64-QAM & $3 / 4$ & $-70 \mathrm{dBm}$ \\
\hline
\end{tabular}


Several rate adaptation mechanisms for multicast in WLANs have been introduced in the literature [13-15]. To apply those mechanisms, the transmitter needs to obtain feedback from multicast receivers by using additional media access control (MAC)-level signaling methods. In [13], Choi et al. proposed a leader-based multicast with the auto rate fallback (LM-ARF) protocol that combines the NACK-based ARQ mechanism of a leader-based protocol (LBP) with the rate adaptation mechanism of ARF. Rate adaptive multicast (RAM) [14] and the auto rate selection mechanism for multicast (ARSM) [15] adopt a receiver-based approach similar to RBAR. However, the existing rate adaptation algorithms for multicast cannot guarantee efficient multicast transmission, because they only focus on the worst channel condition node.

\subsection{IEEE 802.11aa}

The demand for reliable multicast has prompted standardization efforts in IEEE 802.11aa. The IEEE 802.11aa task group focuses on the robust streaming of audio/ video transport streams. In IEEE 802.11aa, audio/video streams transmit with robustness and reliability, while at the same time allowing for the graceful and fair coexistence of other types of traffic. IEEE 802.11aa has specified group-addressed transmission service (GATS) and stream classification service (SCS) to support robust streaming of audio/video transport streams.

GATS and SCS are illustrated in Figures 1 and 2. GATS offers directed multicast service (DMS), groupcast with retries (GCR), GCR unsolicited retries (GCR-UR), GCR block ACK (GCR-BA), and legacy no-ACK/no-retry multicast. The DMS converts multicast traffic to unicast frames directed to each of the group receivers. GCR-UR transmits a multicast frame several times. And, GCR-BA extends the block ACK scheme specified in
IEEE 802.11 n, which requests one or more of the recipients to acknowledge receipt of the transmitted frames.

In contrast, SCS differentiates between separate streams within the same access category to allow graceful degradation of a stream in the event of bandwidth shortage. Using GATS and SCS enables IEEE 802.11aa to provide improved reliable multicast transmission due to using fixed low transmission rate. However, IEEE 802.11aa still cannot utilize the IEEE 802.11 multi-rate WLANs capability properly.

\subsection{Fountain code}

Application layer-forward error correction (AL-FEC) such as Luby transform (LT) [16] and Raptor codes [17], also known as fountain codes, is a block-based forward error correction (FEC) scheme. It has many advantages in multicasting owing to its high coding efficiency, low encoding/decoding processing time, and flexibility in heterogeneous receiver conditions. For this reason, fountain codes are applied to standard multimedia communication systems such as multimedia broadcast multicast services (MBMS) within 3rd Generation Partnership Project (3GPP) Universal Mobile Telecommunications System (UMTS) networks [18] and Internet Protocol (IP)-datacast (IPDC) within Digital Video BroadcastingHandheld (DVB-H) networks [19].

An LT code provides complete recovery of a transmitted source block. In the LT code, a data stream is divided into multiple source blocks by a predefined size. Then, an encoding symbol is generated by performing bitwise XOR operations on selected source symbols. An encoding matrix realization can be characterized by the seed number of a pseudorandom number generator. The LT code decoding process is conducted through message passing. The decoder finds an encoding symbol consisting of only a single source symbol. When its source

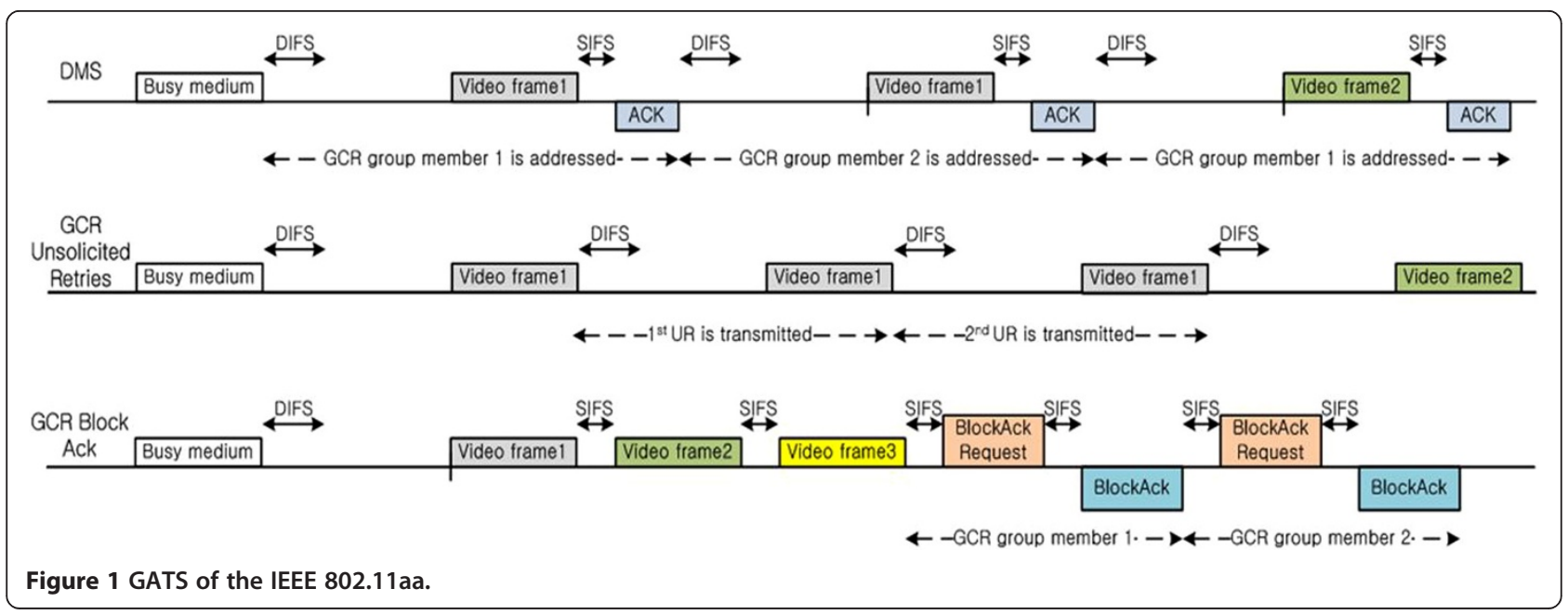




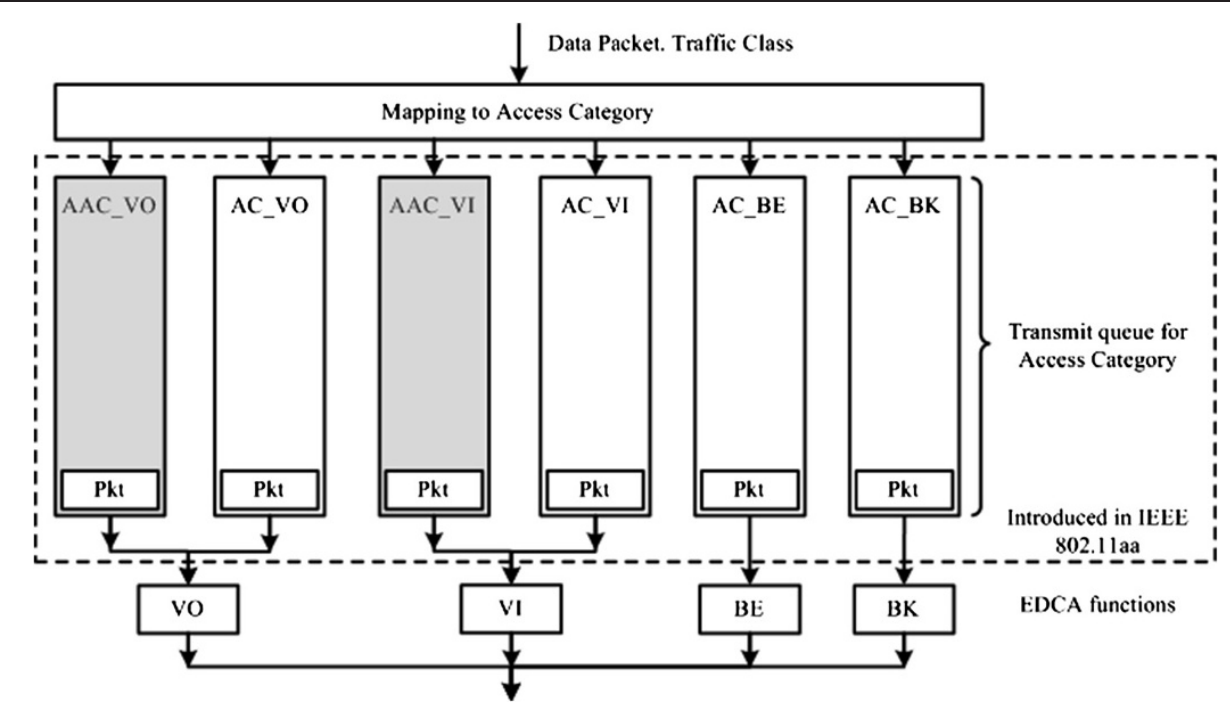

Figure 2 SCS of the IEEE 802.11aa.

symbol is recovered using the corresponding encoding symbol, it is added to all the encoded symbols in which the source symbol is included. In general, the fountain code rate is defined as the number of encoding symbols required for successful fountain decoding, which depends on decoding probability. The LT code encoding/decoding complexity is in the order of $O(k \log k)$ for a source block of length $k$.

Raptor codes are an extension of LT codes that achieve a linear encoding/decoding processing time. A Raptor code is capable of producing an unlimited sequence of encoded symbols (i.e., $n \rightarrow \infty$ ) for a block of $k$ fixedlength source symbols. Such codes are 'rateless' allowing the actual number of encoded symbols and thus the code rate to be determined as needed to combat the current level of network packet loss. For a fixed number $k$ of information symbols, the performance of the Raptor code can be described using three characteristics, the input symbol's erasure probability, the probability of a successful decoding, and the required code rate $k / n$ to achieve target reliability for the given erasure probability. When $k$ information symbols are processed by an encoder, $n-k$ output parity symbols for erasure correction are generated, where the required code rate $k / n$ is decided by the symbol erasure probability and the required reliability for a given $k$. In addition, the symbol erasure probability is a function of PHY layer parameters such as a modulation degree and channel code rate, which are determined by wireless channel quality. That is, if wireless channel quality is known by a transmitter, we can determine $n-k$ overhead symbols to guarantee multimedia service QoS. Moreover, the probability of a successful decoding is related to encoding degree information, namely, the number of initial fragments present in the encoded packet. The Raptor code has encoding/decoding complexity of order $O(k)$.

\subsection{SVC}

Energy-scalable system design [20] involves creating a controllable trade-off between performance and energy consumption, rather than designing a static system. Based on this flexibility, the system can adapt to the dynamic environment and use conditions. SVC is a wellknown energy-scalable system design scheme that has been used widely to address different users with different video scalability. It provides full scalability, including spatial, temporal, and SNR. A scalable bit stream must be organized in such a way that specific content data from the compressed bit stream can be accessed, at different resolutions, without having to decode the full bit stream. This significantly decreases decoding complexity at lower resolution by removing parts of the stream responsible for high-resolution prior decoding. That is, a scalable video stream consists of two types of stream: a base layer stream containing the essential basic data required to reconstruct the most scalable layer of a video and enhancement layers used for the reconstruction of higher scalable levels of the video.

H.264/AVC [21] is a well-known SVC scheme. Also, in H.264/AVC, different types of frames, such as I-, P-, and B-frames can be used by an encoder. An I-frame is a self-contained frame that can be decoded independently without any reference to other images. The first image in a video sequence is always an I-frame. I-frames are 
needed as starting points for new viewers or for resynchronization points, if the transmitted bit stream is damaged. An encoder will automatically insert I-frames at regular intervals or on demand, if new clients are expected to join in viewing a stream. The drawback of I-frames is that they consume many more bits; however, on the other hand, they do not generate many artifacts. A P-frame refers to parts of earlier I- or P-frames to code a frame. P-frames usually require fewer bits than I-frames, but have the drawback of being very sensitive to transmission errors because of their complex dependency on earlier reference P- and I-frames. A B-frame is a frame that refers to both an earlier reference frame and a future frame. When a video decoder restores a video by decoding the bit stream frame by frame, decoding must always begin with an I-frame. P- and B-frames, if used, must be decoded together with the reference frame. Consequently, I- and P-/B-frames differ in importance.

\section{Proposed leader-based multicast scheme with a Raptor code \\ 3.1 Overview}

In this section, we present our leader-based multicast scheme with a Raptor code for efficient multicast transmission in IEEE 802.11 multi-rate WLANs. Functionally, two methods are combined in the proposed scheme. One is a leader selection method and the other is a compensation method.

Typically, leader-based protocols in rate-adaptive multicast schemes for IEEE 802.11 WLANs assign the role of a group leader to the multicast receiver exhibiting the worst signal quality in the group. The group leader is responsible for transmitting an ACK frame of the multicast packets, while other mobile nodes (MNs) may transmit NACK frames when they detect errors. It is a good way to provide reliable multicast transmission to all of multicast receivers but average throughput would be degraded. In contrast to the existing leader-based approaches, in the proposed scheme, we select the group leader node by predefined leader cost (LC) and instead of finding a node having the worst signal quality.

Our new leader selection method may degrade the performance of nodes that are in worse condition than the leader node. We use peak signal-to-noise ratio (PSNR) as the performance metric for multimedia data quality. This is defined by the following formula.

$$
\operatorname{PSNR}=20 \cdot \log _{10}\left(\frac{M_{I}}{\sqrt{\mathrm{MSE}}}\right)
$$

where $M_{I}$ is the maximum possible pixel value of the image and MSE is the mean square error between the original video and impaired videos. In this paper, we use eight bits per sample; hence, $M_{I}$ is set to 255 . MSE is calculated as follows.

$$
\operatorname{MSE}=\frac{1}{m n} \sum_{i=0}^{m-1} \sum_{j=0}^{n-1}[\operatorname{Original}(i, j)-\operatorname{Degraded}(i, j)]^{2}
$$

In a multicast scenario, to guarantee the minimum required QoS, we define PSNR required $_{\text {to }}$ be the minimum required PSNR among the multicast group receivers, as follows.

$\mathrm{PSNR}_{\text {required }}=\operatorname{Min}\left(\mathrm{PSNR}_{\text {required_1 }}, \mathrm{PSNR}_{\text {required_2 }}, \ldots, \mathrm{PSNR}_{\text {required } \_n}\right)$

In the proposed scheme, performance degradation of nodes that are in worse condition than the leader node is compensated with a Raptor code and SVC with UEP.

In particular, the proposed scheme consists of an SVC block with UEP for graceful degradation, a leader selection block, and a link adaptation block with the Raptor code. The leader selection block and the link adaptation block are operated at the multicast transmitter (MT), while the SVC block with UEP is operated at both MT and multicast receiver nodes. An overview of the proposed scheme is illustrated in Figure 3. Real-time transport protocol (RTP), UDP, IP, and IEEE 802.11 logical link control (LLC) are used to transmit multimedia traffic. The detailed operations of each block are described in the following subsections.

\subsection{SVC block with UEP}

In the SVC with UEP block, video streams are first encoded by base and enhancement layers. The base layer stream containing the essential basic data is required to reconstruct the most scalable layer of the video and determines the minimum required QoS. In addition, several enhancement layers for the reconstruction of higher scalable levels of the video are encoded.

All multicast group nodes must be guaranteed to receive the base-layer stream. Reception of the enhancement layer streams is achieved selectively according to each receiver's channel state. If a particular node cannot decode the base-layer stream, it transmits a NACK frame to require a change of the transmission rate. Additionally, we use a UEP scheme based on encoding degree information by the frame type. In general, an I-frame is more important than a B- or P-frame. Thus, when the Raptor code overhead is determined according to a channel state, we assign a high degree value of a Raptor code to an I-frame. The encoded degree of the Raptor code corresponds to the number of initial fragments present in the encoded packet. We define four degree encoding patterns, described in Table 2. 

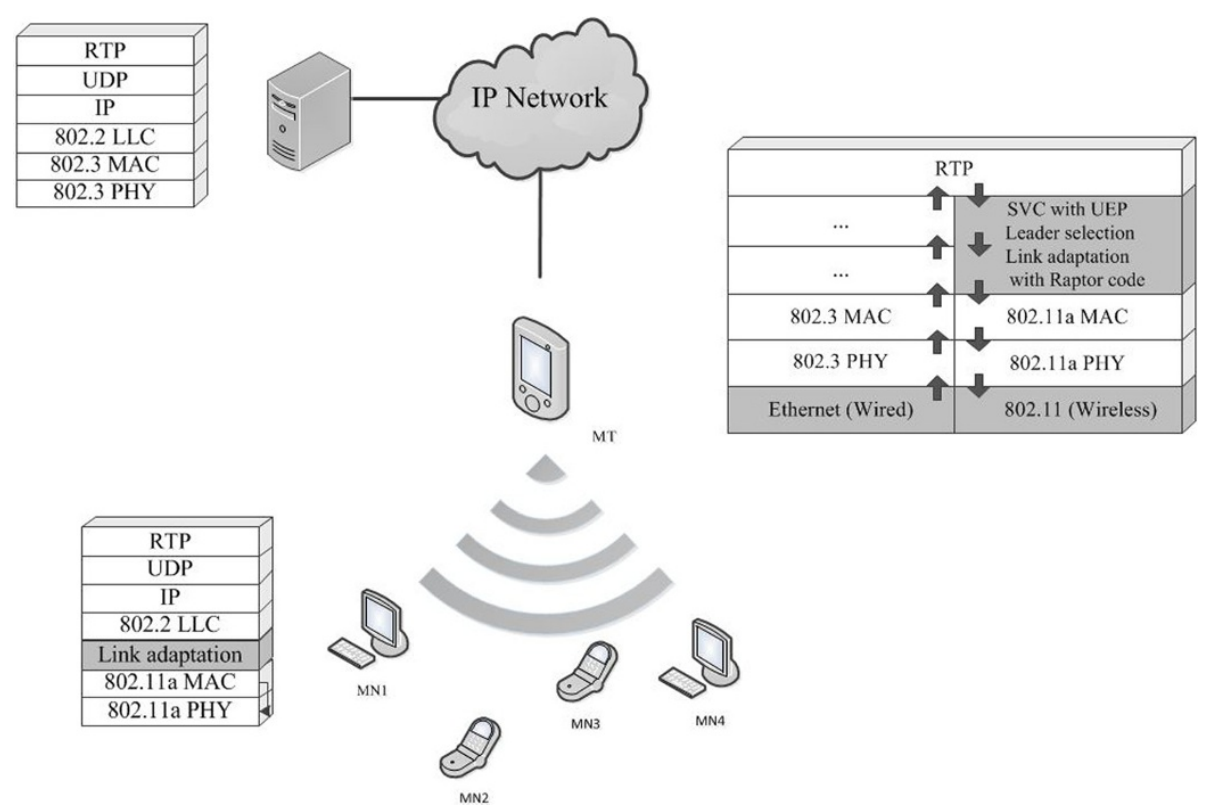

Figure 3 The overview of the proposed scheme in IEEE 802.11 multi-rate WLANs.

The pattern used in an encoded packet is transmitted through a reserved field of the IP header. In this paper, the pattern is pre-determined by the user and the mandatory pattern is pattern two. In addition, pattern four means no use of the Raptor code nor UEP scheme, pattern three means use of the Raptor code without the UEP scheme, and pattern one means use of the Raptor code only for I-frame protection. Consequently, being assigned with a high degree value, the I-frame is transmitted relatively several times, so the I-frame is more protected than the B- and P-frames.

\subsection{Leader selection block}

MT records the received signal strength (RSS) of each $\mathrm{MN}$ and the valid time by monitoring previous transmissions for the MN. To record the channel state of MNs, the MT manages a channel state table (CST). The CST consists of three entities, MN ID, RSS_mode, and record time (RT). In this paper, RSS mode is mapped to PHY mode.

When multicast traffic is delivered to the MT, the MT selects a multicast group leader among the multicast group members by calculating the LC. If there is no change in wireless channel, all nodes can be considered to be fixed; we can define $\mathrm{LC}$ as below

$$
\text { LC }=\text { RSS_mode }
$$

However, if we want to consider changes in the wireless channel, the current value of LC of each node could be invalid since it is possible that the channel state has already been changed. Therefore, we add a valid time to the $\mathrm{LC}$ value so changes in the wireless channel condition can be reflected in $\mathrm{LC}$.

$$
\text { LC }=\text { RSS_mode }+ \text { valid time }
$$

valid time $=$ record time + coherence time-current time

where record time refers to the moment that RSS_mode is changed, coherence time is the time duration over which the channel impulse response is considered to be invariant, and current time is the time instant at which transmission is started. Thus, the valid time represents how much time remains from the current time to the end of the coherence time.

Table 2 Priority based degree encoding information

\begin{tabular}{llll}
\hline Pattern & I-frame & B-frame & P-frame \\
\hline 1 (Using both Raptor code and UEP) & Degree 4 & Degree 1 & Degree 1 \\
2 (Using both Raptor code and UEP) & Degree 3 & Degree 2 & Degree 1 \\
3 (Using a Raptor code but not UEP) & Degree 2 & Degree 2 & Degree 2 \\
4 (Neither using a Raptor code nor UEP) & Degree 1 & Degree 1 & Degree 1 \\
\hline
\end{tabular}


A valid time can be relatively small or large, depending on RSS_mode. Valid time with RSS mode eight is eight times larger than its value with RSS mode one. So we gave a weighted value of RSS_mode to valid time.

$$
\text { LC }=\text { RSS_mode }+ \text { RSS_mode } \cdot \text { valid time }
$$

Consequently, we rewrote the above equation with weighting factor $\alpha$ as below

$$
\mathrm{LC}=\mathrm{RSS}_{\text {mode }}+\alpha \cdot \mathrm{RSS}_{\text {mode }} \cdot \text { valid time }
$$

where $\alpha$ is a weighting factor between the first term (RSS_mode) and the second term (RSS_mode - valid time). The value of $\alpha$ changes as the network channel state alters, and we simulate PSNR performance according to $\alpha$. As seen in Figure 4, we can check the optimal $\alpha$ value depending on the channel state. In the case that a node moves fast, increasing the value of $\alpha$ for the second term can show a better performance.

After calculating LC, the MT selects a leader node among the multicast group members. As we mentioned in Section 3.1, we select a node having the median LC value (average channel condition node) as the leader node, rather than a node having the worst $\mathrm{LC}$ value (worst channel condition node).

We propose a leader selection method that iterates until all multicast receivers satisfy the minimum required PSNR, and we use the median LC value node as the initial point in iterative leader selection, which is an efficient and simple solution. Table 3 shows the average convergence time for each initial leader selection point. Consequently, link adaptation operates between the MT and average channel condition node. If a particular node cannot satisfy the minimum required QoS, it sends a NACK frame to the MT. When the MT does not receive an ACK frame
Table 3 Average leader selection convergence time

\begin{tabular}{ll}
\hline Leader selection rule & $\begin{array}{l}\text { Average convergence } \\
\text { time (iteration number) }\end{array}$ \\
\hline Best channel state node & \\
$\begin{array}{l}\text { Next leader selection: select a best channel } \\
\text { state node without previously determined }\end{array}$ & 5.4 \\
leader node & 0.8 \\
$\begin{array}{l}\text { Median channel state node } \\
\text { Arithmetic average channel state node }\end{array}$ & \\
$\begin{array}{l}\text { Next leader selection: select an arithmetic } \\
\text { average channel state node without } \\
\text { considering previously determined } \\
\text { leader node }\end{array}$ & \\
\hline
\end{tabular}

within timeout or receives a NACK frame, it adopts more reliable PHY modes using the link adaptation scheme with the Raptor code or selects a new leader node among the multicast group members. The link adaptation scheme is described in detail in section 3.4.

\subsection{Link adaptation block with a Raptor code}

MT transmits multicast data packets at a proper transmission rate selected by the link adaptation scheme. In the proposed scheme, the signal strength of the leader's ACK frame is used as the channel estimation metric. When the MT transmits a multicast data packet, it initiates a timer and waits for the leader's ACK frame. If an ACK frame is received before timeout, the MT thinks that the previous transmission is completed and calculates the received ACK frame's signal strength. The MT chooses the proper transmission rate for the next transmission based on the ACK frame's signal strength.

In the proposed scheme, the proper PHY mode (transmission rate) and frame length are determined by the

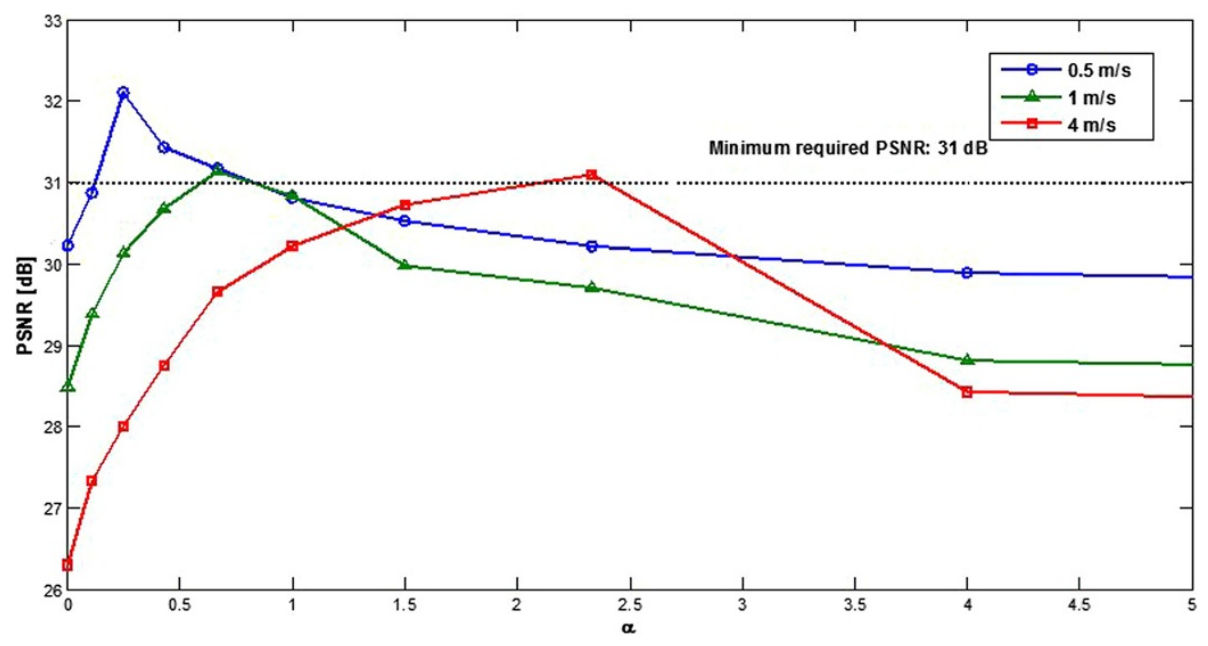

Figure 4 PSNR performance vs. a. 
leader's ACK signal strength and are stored in a transmission matching table (TMT), a pre-computed lookup table with the best PHY mode and frame length for each RSS. In addition, after determining the frame error rate of the MAC layer by using TMT, we select the proper Raptor code overhead. This relation is described in Figure 5.

If the data frame is received successfully, the multicast group leader transmits an ACK frame after a short interframe space (SIFS) to the MT. Other multicast group nodes do not transmit an ACK frame to the MT. Only multicast group nodes that are not satisfied with the minimum required QoS (i.e., required frame error rate) transmit NACK frames to the MT after SIFS. Thus, when the ACK frame from the multicast group leader and NACK frames from specific multicast group nodes are transmitted concurrently, those ACK and NACK frames will collide at the MT. If the MT does not receive a leader's ACK frame within timeout or receives a multicast member's NACK frame, the MT adjusts the transmission rate or selects a new leader.

If the MT judges that the transmission is failed, then it adjusts the transmission rate to satisfy the required QoS level. In this case, the MT selects the next transmission rate by NACK frame signal strength or transmits by the next reliable transmission rate. However, when the event of a NACK frame received or an ACK frame not received within timeout occurs twice consecutively even though the MT has changed the transmission rate, the MT decides that the current leader node is not suitable to represent other multicast receivers any more. Thus, a leader node reselection procedure is performed as follows. In the reselection procedure, LC is re-calculated and the 'median - $i$ th' value node is selected. $i$ is the number of consecutive iteration of the reselection procedure according to transmission failure or success. When multicast transmissions succeed ten times, the MT invokes the leader node reselection procedure to enhance throughput. In this case, we select the 'median $+i$ th' value node as the leader node. Figure 6 shows the proposed protocol timing.

In the proposed scheme, both a Raptor overhead table (ROT) and TMT are used for enhancing throughput while satisfying the minimum required QoS. The ROT consists of a symbol length, Raptor overhead, and expected received symbol erasure probability. Shown in Equation 9, we modified the expected received symbol erasure probability of the Raptor code based on [22]. The Raptor code performance is shown in Figure 7. In Figure 7, the Raptor code performance is represented by the Raptor code overhead.

Given the expected received symbol erasure probability, the output frame error probability of the application layer can be calculated as

$$
P_{f}\left(n, P_{e}\right)=0.85 \times 0.567^{n-k-P_{e} \cdot n}
$$

where $n$ is the Raptor codeword length, $k$ is the input source symbol length, and $P_{e}$ is the input symbol erasure probability in the application layer. Also, $P_{e}$ is described as output frame error rate in the MAC layer. $P_{e}$ is determined by the PHY modulation scheme, PHY channel coding rate, and MAC frame length at given SNR. Under the assumption of binary convolutional coding and hard-decision Viterbi decoding in the PHY layer, $P_{e}$ can be calculated as

$$
P_{e}^{m}(L) \leq 1-\left(1-P_{u}^{m}\right)^{8 L}
$$

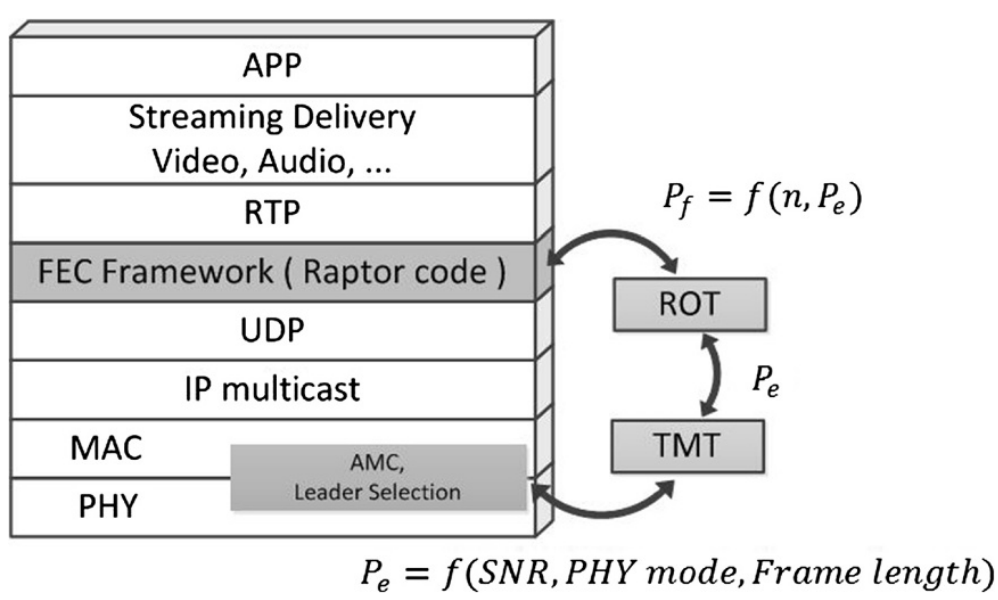

Figure 5 Protocol stack. 

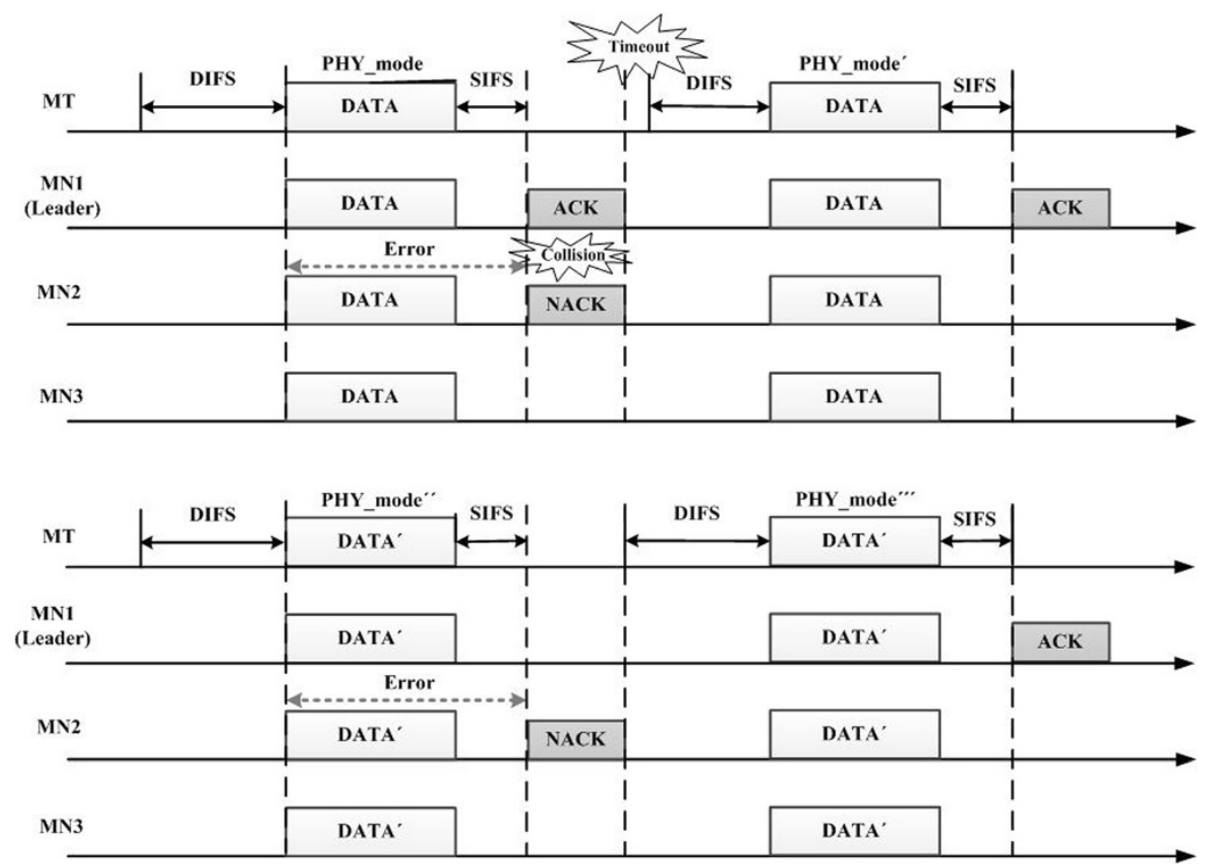

Figure 6 Protocol timing.

where $m$ is the transmission mode (PHY mode) and $L$ is the frame length. The union bound $P_{u}^{m}$ of the first-event error probability is given by

$$
P_{u}^{m}=\sum_{d=d_{\mathrm{free}}}^{\infty} a_{d} \cdot P_{d}
$$

where $d_{\text {free }}$ is the free distance of the convolutional code, which is the PHY FEC code (PHY channel code), selected in PHY mode $m, a_{d}$ is the total number of error events of weight $d$, and $P_{d}$ is the probability that an incorrect path at distance $d$ from the correct path is being chosen by the Viterbi decoder.

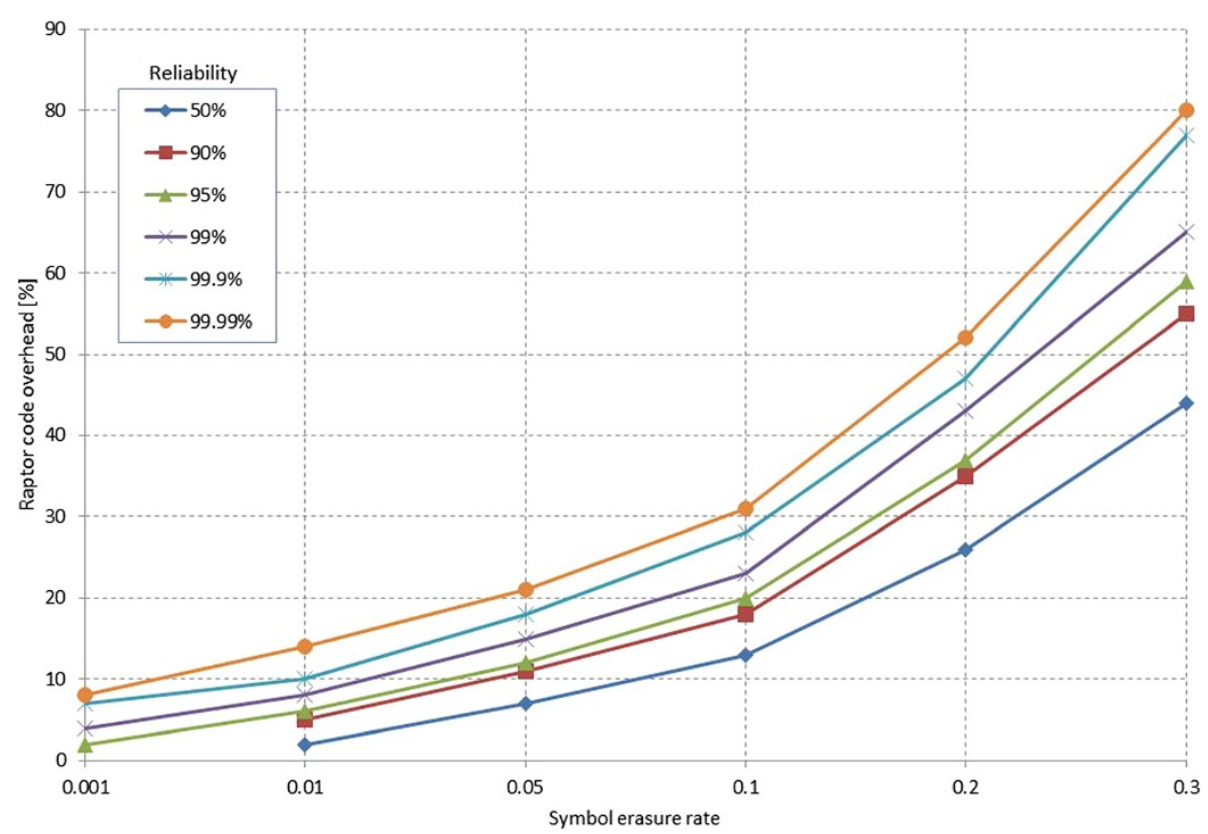

Figure 7 Symbol erasure rate vs. Raptor code overhead for probability of successful decoding (reliability). 
When hard decision decoding is applied, $P_{d}$ is given by

$$
P_{d}= \begin{cases}\sum_{k+1}^{d}\left(\begin{array}{l}
d \\
k
\end{array}\right) \cdot \rho^{k} \cdot(1-\rho)^{d-k}, & \text { if } d \text { is odd } \\
\frac{1}{2} \cdot\left(\begin{array}{l}
d \\
\frac{d}{2}
\end{array}\right) \cdot \rho^{\frac{d}{2}} \cdot(1-\rho)^{\frac{d}{2}}+\sum_{k=\frac{d}{2}+1}^{d}\left(\begin{array}{l}
d \\
k
\end{array}\right) \cdot \rho^{k} \cdot(1-\rho)^{d-k}, & \text { if } d \text { is even }\end{cases}
$$

where $\rho$ is the bit error probability for the modulation scheme selected in PHY mode $m$, and the detailed derivation of $\rho$ is presented in [23]. The value of $a_{d}$ can be obtained by a numerical search [24].

From Equations 9 and 12, we can determine the proper Raptor code overhead for a given input symbol erasure probability. Thus, the TMT consists of four input parameters - channel SNR value, PHY modulation scheme, PHY channel coding rate, and MAC frame length - and one output parameter - input symbol erasure probability.

Consequently, we can jointly estimate the optimal Raptor code overhead, PHY modulation scheme, PHY channel coding rate, and MAC frame length using ROT and TMT given the current SNR. We summarize our link adaptation scheme as follows:

1. Check the current channel SNR from the leader's ACK or explicit NACK information.

2. Calculate the expected $P_{e}$ from the current PHY modulation scheme, PHY channel coding rate, and MAC frame length to enhance the network throughput using TMT.

3. Calculate the Raptor code overhead by using ROT.

\section{Performance evaluation}

In this section, we evaluate the effectiveness of the proposed leader-based multicast scheme with a Raptor code. To evaluate the proposed scheme, the source video is encoded in H.264/AVC format using the Joint Scalable Video Model (JSVM), the reference software for H.264/SVC coding/decoding released and maintained by the MPEG/ITU Joint Video Team [25].

First, a YUV video is encoded in the H.264/SVC format using the JSVM encoder. In this procedure, a Raptor code with the UEP scheme is added. Then, in an ns-2 simulated environment, an MT transmits the encoded video to multicast receivers through several multicast protocols. Each multicast receiver stores received video data and decodes the data into three layers: a base layer, enhancement layer1, and enhancement layer2, in JSVM. In addition, we use the scalable video coding streaming evaluation framework (SVEF)
Table 4 Convert table from PSNR to MOS

\begin{tabular}{llll}
\hline MOS & Quality & Impairment & PSNR (dB) \\
\hline 5 & Excellent & Imperceptible & $>37$ \\
4 & Good & Perceptible but not annoying & $31 \sim 37$ \\
3 & Fair & Slightly annoying & $25 \sim 31$ \\
2 & Poor & Annoying & $20 \sim 25$ \\
1 & Bad & Very annoying & $<20$ \\
\hline
\end{tabular}

[26] for error concealment of decoded video. For ns-2 simulations, we set up an IEEE 802.11a WLAN consisting of one MT with different number of receivers. In the first scenario, multicast receivers are fixed, while in the second scenario, multicast receivers move at $1 \mathrm{~m} / \mathrm{s}$ following a random waypoint model.

We use mean opinion score (MOS) to provide a numerical indication of the perceived video quality as the minimum required QoS level. As shown in Table 4, MOS is indicated using a score of five steps, and PSNR can be converted to MOS based on ITU-R BT. 500-11 [27].

In this paper, we assign the MOS value of four as the minimum required QoS level. That is, the proposed scheme aims to enhance the throughput while maintaining PSNR above $31 \mathrm{~dB}$. The throughput is measured at the MT and is represented as system throughput. Furthermore, we measure the throughput at the MAC layer, because the proposed scheme aims for throughput enhancement by using link adaptation in good channel conditions.

First, we have checked the PSNR performance in patterns, as described in Table 2. Figure 8 shows the PSNR performance per pattern. Points on the X-axis of $-89,-85,-78 \mathrm{dBm}$ are RSS_mode changing points; hence, they have a high transmission error probability. As seen in Figure 8, we can check the coding gain of the Raptor code by comparing patterns 3 and 4 . In addition, the UEP gain is almost $3.1 \mathrm{~dB}$, which is confirmed by comparing patterns 2 and 3 . And we checked that patterns 3 and 4 have a better PSNR performance than the

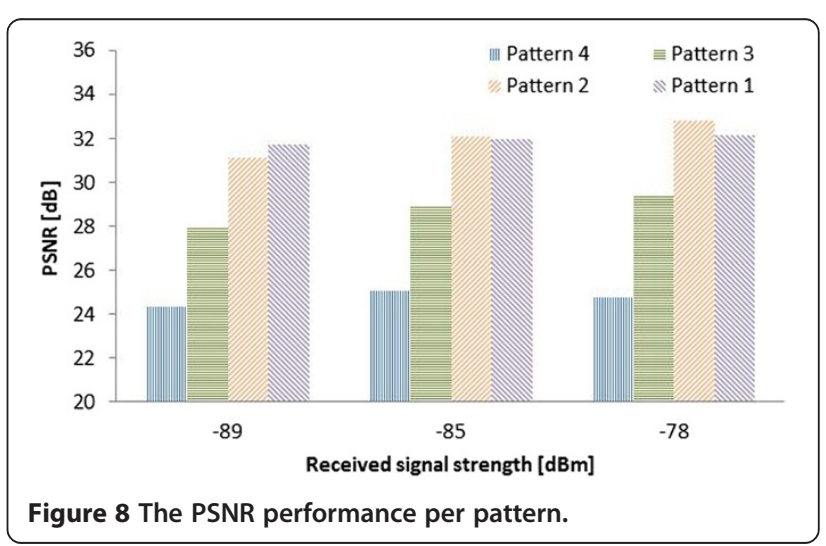




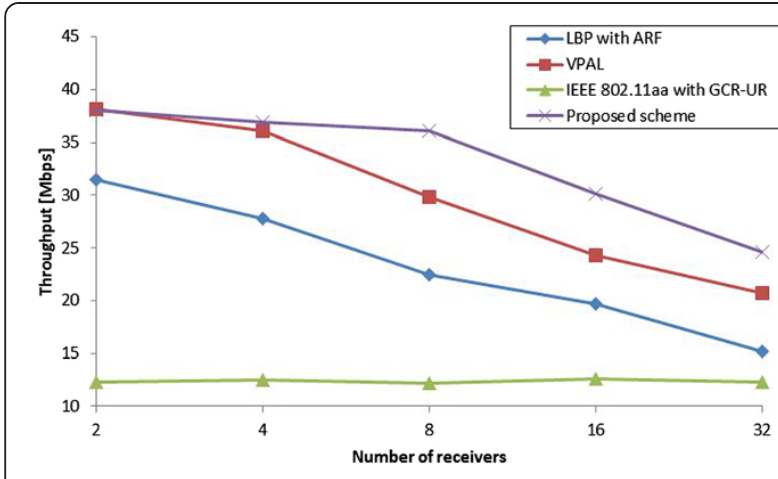

Figure 9 Comparison of throughput between the proposed scheme and existing schemes in fixed network.

other patterns. Furthermore, we checked that pattern 4 has a better PSNR performance than pattern 3 in a bad channel condition. However, pattern 3 which protects both I- and B-frames outperforms pattern 4 as channel state becomes better.

Then, we compared the proposed scheme with LBP with ARF [13], VPAL [10], and IEEE 802.11aa with GCR-UR [2].

Figures 9 and 10 show a comparison of throughput between the proposed scheme and existing schemes with different numbers of receivers in fixed and mobile networks, respectively. In Figure 9, all schemes except the IEEE 802.11aa suffer degradations of throughput as the number of receivers increases due to collision by contending nodes and increased frame errors with interference from neighboring nodes. However, the IEEE 802.11aa with the GCR-UR scheme is not affected by the numbers of receivers, and it has the lowest throughput, because it always uses a fixed and reliable transmission regardless of wireless channel condition. And, the proposed scheme is still much more efficient than other schemes. Because the link adaptation techniques of the

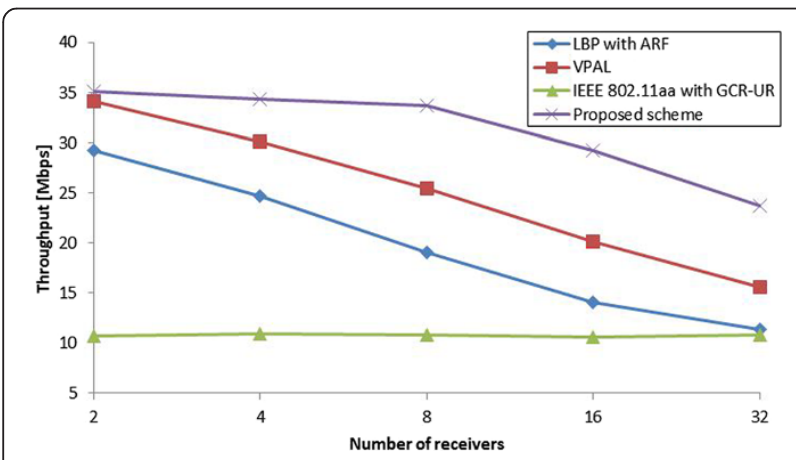

Figure 10 Comparison of throughput between the proposed scheme and existing schemes in mobile network (mobility with $1 \mathrm{~m} / \mathrm{s}$ ).

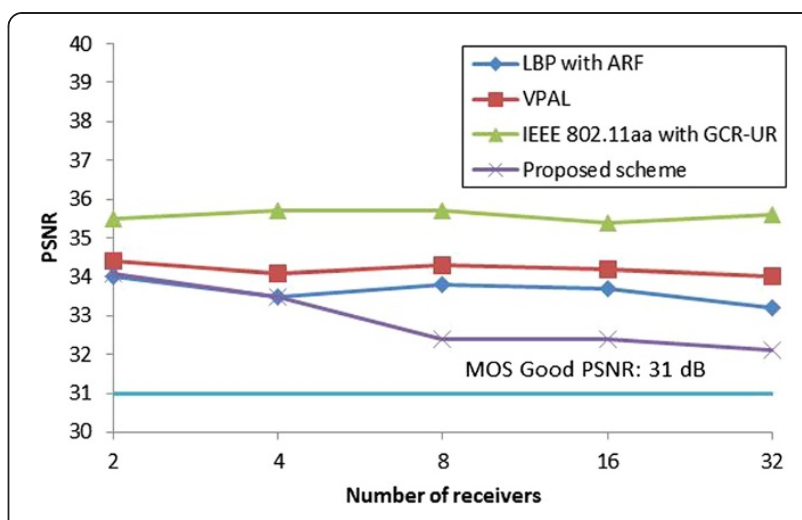

Figure 11 Comparison of PSNR between the proposed scheme and existing schemes in fixed network.

LBP with ARF and VPAL scheme are focused on the worst channel condition node, both schemes cannot utilize wireless channel capacity properly. In contrast, the proposed scheme is focused on a specific leader node selected by using LC; hence, it can utilize wireless channel capacity efficiently. In Figure 10, we check the throughput performance in mobile network. As expected, the proposed scheme still has the best throughput performance among other existing schemes. However, overall performance is degraded by mobility which leads worse wireless channel condition than fixed one.

Figures 11 and 12 represent comparison of the worst PSNR between the proposed scheme and existing schemes with different numbers of receivers in fixed and mobile networks, respectively. As seen in Figure 11, the IEEE 802.11aa has the best PSNR performance due to its robust transmission characteristics. And the VPAL scheme has the better performance than the LBP with ARF scheme due to its Raptor coding gain. Unfortunately, the proposed scheme has the lowest PSNR performance because

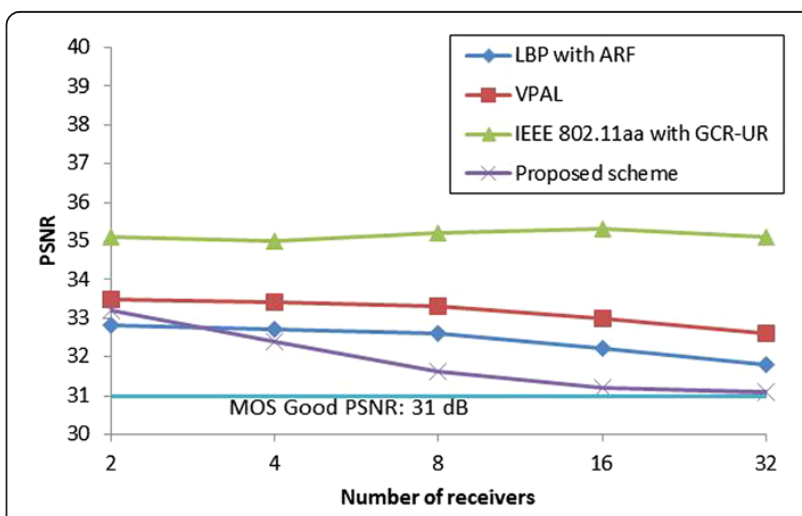

Figure 12 Comparison of PSNR between the proposed scheme and existing schemes in mobile network (mobility with $1 \mathrm{~m} / \mathrm{s}$ ). 
Table 5 Performance comparison through various images

\begin{tabular}{|c|c|c|c|c|c|}
\hline & & \multicolumn{2}{|l|}{ Fixed } & \multicolumn{2}{|l|}{ Mobile } \\
\hline & & Worst PSNR (dB) & Throughput (Mbps) & Worst PSNR (dB) & Throughput (Mbps) \\
\hline \multirow[t]{4}{*}{ Foreman [25] } & LBP with ARF & 33.7 & 21.1 & 32.2 & 16.1 \\
\hline & VPAL & 34.2 & 26.3 & 33.1 & 23.7 \\
\hline & IEEE 802.11aa with GCR & 35.6 & 12.3 & 35.3 & 10.7 \\
\hline & Proposed scheme & 32.4 & 33.6 & 31.5 & 31.9 \\
\hline \multirow[t]{4}{*}{ Crew [25] } & LBP with ARF & 33.1 & 20.9 & 31.9 & 16.7 \\
\hline & VPAL & 33.8 & 26.1 & 32.6 & 23.4 \\
\hline & IEEE 802.11aa with GCR & 35.1 & 12.2 & 34.9 & 10.9 \\
\hline & Proposed scheme & 31.8 & 33.1 & 31.2 & 30.9 \\
\hline \multirow[t]{4}{*}{ Soccer [25] } & LBP with ARF & 33.3 & 21 & 32 & 16.4 \\
\hline & VPAL & 33.9 & 26.1 & 32.9 & 23.5 \\
\hline & IEEE 802.11aa with GCR & 35.3 & 12.3 & 35.1 & 10.8 \\
\hline & Proposed scheme & 31.9 & 33.4 & 31.1 & 31.4 \\
\hline
\end{tabular}

particular nodes having poor wireless channel condition than the leader node can undergo performance degradation. However, in the proposed scheme, those nodes are compensated by the SVC with UEP and the Raptor code; hence, they can be guaranteed to satisfy the minimum required QoS level which is over $31 \mathrm{~dB}$ PSNR. Also, in Figure 12, the proposed scheme still satisfies the minimum required QoS level in mobile network. The PSNR performance corresponding to the schemes in $[10,13]$, and the proposed scheme in mobile network are slightly lower than those in the fixed network due to a worse channel state caused by node mobility. Especially, the IEEE 802.11 aa with the GCR scheme still has similar PSNR performance regardless of node mobility and number of nodes, because the scheme always sends a reliable transmission rate and uses a retransmission scheme.

Additionally, we evaluate the throughput and the worst PSNR performances with different image files which are referred in [25]. Table 5 summarizes the throughput and the worst PSNR performance through various images. In this simulation, multicast receivers are fixed to ten, and other parameters are the same to previous simulation parameters. As we can check in Table 5, the IEEE 802.11aa with GCR-UR has the best PSNR performance regardless of image files. Unfortunately the proposed scheme has the lowest PSNR performance among existing schemes; however, the PSNR of the proposed scheme is still over $31 \mathrm{~dB}$ which is a perceptible PSNR value. On the other hand, we confirm that the throughput performance of the proposed scheme is better than that of other existing schemes. Consequently, we demonstrate that the proposed scheme enhances the throughput while guaranteeing the minimum required PSNR by comparing to existing schemes through various images.

\section{Conclusions}

We propose a new leader-based multicast scheme with a Raptor code for efficient multicasting in IEEE 802.11 multi-rate WLANs. The proposed scheme consists of three blocks: a leader selection block, an SVC block with UEP, and a link adaptation block with the Raptor code. The leader selection block chooses a leader node which can enhance the link utilization. PSNR degradation which is caused by the leader selection rule is compensated by the SVC with UEP block and the Raptor code block. Particularly, for each packet transmission, the MT selects the Raptor code overhead, MAC frame length, and PHY mode by using a simple table-based approach in order to suit the given wireless channel condition. Further, an additional UEP scheme that protects Iframes more than B- and P-frames is used to guarantee a specific QoS requirement. We show that the proposed scheme has a better throughput while guaranteeing the minimum required QoS compared to existing multicast schemes

\section{Competing interests}

The authors declare that they have no competing interests.

\section{Acknowledgements}

This work was supported partially by the National Research Foundation of Korea (NRF) grant funded by the Korea government (MEST) (No. NRF2012K1A3A1-A09026959).

\section{Author details}

${ }^{1}$ The School of Electrical Engineering, Korea University, Anam-dong, Seongbuk-Gu, Seoul 136-701, South Korea. ${ }^{2}$ The School of Communication Engineering (Sup'Com) Techno Park EL Ghazala 2083, Ariana, Tunisia.

Received: 30 December 2013 Accepted: 28 October 2014 Published: 11 November 2014 


\section{References}

1. IEEE, IEEE P802.11ac. Specification framework for TGac. IEEE 802.11-09/0992r21 (IEEE, Piscataway, 2011)

2. IEEE, IEEE TGaa, Draft P802.11aa D0.0.3, Amendment 8: MAC enhancements for Robust Audio Video Streaming (IEEE, Piscataway, 2010)

3. A Harris, G Ireland, Enabling IPTV: What carriers need to know to succeed (International Data Corporation, Ireland, 2005). White paper

4. IEEE, IEEE Standard for Information technology-Telecommunications and information exchange between systems Local and metropolitan area networks-Specific requirements Part 11: Wireless LAN Medium Access Control (MAC) and Physical Layer (PHY) Specifications. IEEE std802.11-1999 (IEEE, Piscataway, 1999), pp. 1-528

5. J Kuri, SK Kasera, Reliable multicast in multi-access wireless LANs. Proc. INFOCOM'99, vol. 2 (New York, 1999), pp. 760-767

6. Y Park, Y Seok, N Choi, Y Choi, J-M Bonnin, Rate-adaptive multimedia multicasting over IEEE 802.11 wireless LANs, in Proceedings of the 3rd IEEE Consumer Communications and Networking Conference (CCNC 2006) (IEEE, Las Vegas, 2006)

7. A Basalamah, H Sugimoto, T Sato, Rate adaptive reliable multicast MAC protocol for WLANs, in Proceedings of the 63rd IEEE Vehicular Technology Conference (VTC 2006-Spring) (IEEE, Melbourne, 2006)

8. J Villalon, P Cuenca, LO Barbosa, Y Seok, T Turletti, Cross-layer architecture for adaptive video multicast streaming over multirate wireless LANs. IEEE Trans. Selec Areas Commun 25(4), 699-711 (2007)

9. W Wenger, YK Wang, T Schierl, Transport and signaling of SVC in IP network. IEEE Trans. Circuits Syst. Video Technol 17(9), 1164-1173 (2007)

10. M Choi, M Samokhina, K Moklyuk, S Choi, J Heo, SJ Oh, VPAL: video packet adaptation layer for reliable video multicast over IEEE 802.11n WLAN. Comput Commun. 33(18), 2271-2281 (2010). doi:10.1016/j.comcom.2010.06.018

11. A Kamerman, L Monteban, WaveLAN II: A high-performance wireless LAN for the unlicensed band. Bell Labs. Tech. J. 2(3), 118-133 (1997)

12. G Holland, N Vaidya, P Bahl, A rate-adaptive MAC protocol for multi-hop wireless networks, in Proceeding of ACM MOBICOM'01 (ACM, Rome, 2001)

13. S Choi, N Choi, Y Seok, T Kwon, Y Choi, Leader-based rate adaptive multicasting for wireless LANs, in Proceedings IEEE GLOBECOM (IEEE, Washington, 2007)

14. A Basalamah, $\mathrm{H}$ Sugimoto, $\mathrm{T}$ Sato, A rate adaptive multicast protocol for providing MAC layer reliability in WLANs. IEICE Trans. Commun. 3(10), 1216-1220 (2006)

15. J Villalon, P Cuenca, L Barbosa, Y Seok, T Turletti, Cross-layer architecture for adaptive video multicast streaming over multirate wireless LANs. IEEE J. Select. Areas Commun. 25(4), 699-711 (2007)

16. M Luby, LT codes, in Proceedings 43rd Annu (IEEE Symp, Foundations of Computer Science (FOCS) (IEEE, Vancouver, 2002)

17. A Shokrollahi, Raptor codes. IEEE Trans. Inf. Theory 52(6), 2551-2567 (2006)

18. ETSI, Universal Mobile Telecommunications System (UMTS): Mobile Broadcast/ Multicast Service (MBMS): Protocols and Codecs (ESTI, Sophia-Antipolis Cedex, 2005), pp. 1-134

19. ETSI, DigitalVideo Broadcasting (DVB): Transmission System for Handheld Terminals (DVB-H) (ESTI, Sophia-Antipolis Cedex, 2004), pp. 1-14

20. A Sinha, A Wang, A Chandrakasan, Energy scalable system design. IEEE Trans Very Large Scale Integration (VLSI) Syst 10(2), 135-145 (2002)

21. ITU-T. Recommendation, Draft ITU-T recommendation and final draft international standard of joint video specification (ITU-T Rec. H.264/ISO/IEC 14 496-10 AVC, in Joint Video Team (JVT) of ISO/IEC MPEG and ITU-T VCEG, JVTG050 (ITU-T, Geneva, 2003)

22. M Luby, M Watson, T Gasiba, T Stockhammer, Mobile data broadcasting over MBMS tradeoffs in forward error correction, in MUM'06: Proceedings of the 5 th international conference on Mobile and ubiquitous multimedia (ACM, New York, NY, USA, 2006), p. 10

23. D Qiao, S Choi, KG Shin, Goodput analysis and link adaptation for IEEE 802.11a wireless LANs. IEEE Trans. on Mobile Comput (TMC) $1(4), 278-292(2002)$

24. D Haccoun, G Begin, High-rate punctured convolutional codes for Viterb and sequential decoding. IEEE Trans Commun 37(11), 1113-1125 (1989)
25. Video Test Sequence Database. ftp://ftp.tnt.uni-hannover.de/pub/svc/ testsequences/ (2003), Accessed 30 Dec 2013

26. SVEF, Scalable Video-Streaming Evaluation Framework, 2007. http://svef. netgroup.uniroma2.it, Accessed 30 Dec 2013

27. ITU-R. Recommendation, 500-11, Methodology for the subjective assessment of the quality of television pictures (ITU-R, Geneva, 2002)

doi:10.1186/1687-1499-2014-184

Cite this article as: Kim et al:: A new leader-based multicast scheme with a Raptor code in IEEE 802.11 multi-rate WLANs. EURASIP Journal on Wireless Communications and Networking 2014 2014:184.

\section{Submit your manuscript to a SpringerOpen ${ }^{\odot}$ journal and benefit from:}

- Convenient online submission

- Rigorous peer review

- Immediate publication on acceptance

- Open access: articles freely available online

- High visibility within the field

- Retaining the copyright to your article

Submit your next manuscript at $>$ springeropen.com 